\title{
LA ESCUELA PRIMARIA: UNA PERSPECTIVA ETNOGRAFICA*
}

\author{
Por Araceli de Tezanos de Mañana**
}

\section{Presentación}

El proyecto "Escuela y Comunidad en América Latina" se enmarca dentro de una nueva perspectiva de la investigación educativa.

Después de varios meses de trabajo de campo, de reflexiones sobre las informaciones recogidas, de discusiones teóricas al interior del equipo de investigación y con algunos colegas del Centro de Investigaciones, queremos mostrar en este artículo algunos aspectos teóricos y prácticos de esta nueva perspectiva: la etnografía en educación.

En consecuencia, vamos a iniciar este breve ensayo dando una visión general sobre las concepciones en etnografía; para en una segunda instancia hacer un recuento sobre la experiencia de investigación que estamos realizando en nuestro proyecto, dentro de este mareo. Finalmente vamos a referirnos a uno de los que creemos problemas fundamentales en este tipo de estudio: la interpretación de los datos recogidos en las observaciones y entrevistas que llevamos a cabo.

Es necesario aclarar que, en relación a este último punto, lo que presentamos es una primera reflexión sobre las posibles formas y contenidos de la interpretación, a través de la presentación de notas de campo ampliadas con una aproximación inicial de análisis.

El carácter aproximativo se debe a los procedimientos metodológicos que sustentan y estructuran el proyecto.

\section{La etnografía: una alternativa para la investigación educativa.}

La idea de recuperar los procedimientos etnográficos, provenientes de la Antropología, para la investigación educativa, surgió en Estados Unidos a comienzos de los años 70, después de analizar y verificar que los resultados de las investigaciones realizadas por sociólogos, sicólogos y economistas de la Educación, no podían ser aplicados para solucionar los conflictos que se desarrollaban al interior de la instituciones educativas, en todos sus niveles. "A pesar de los millones de dólares que han sido invertidos en investigación educativa durante las últimas dos décadas, esta inversión no ha traído un progreso sustancial en la solución de problemas educativos. No es sorprendente entonces que exista una creciente evidencia de insatisfacción con las metodologías y marcos conceptuales que han guiado la investigación hasta ahora". (Wilcox, K. 1980).

Surge como respuesta a este planteamiento el interés por estudios de tipo cualitativo, en pequeña escala y de profundidad. Estas condiciones son propias a los estudios etnográficos.

\footnotetext{
* Este artículo fue redactado con la colaboración de Guillermo Muñoz, y Emiliano Romero. Investigadores del Centro de Investigaciones de la Universidad Pedagógica Nacional C.I.U.P.

${ }^{* * *}$ Lic. Ciencias de la Educación, Instituto Magisterial Superior, Montevideo, M.A. Investigación Ciencias de la Educación, México. Investigadora Centro de Investigaciones, Universidad Pedagógica Nacional, CIUP.
} 


\section{A. La etnografía: contenidos y procesos.}

Existe el convencimiento que etnografía es sinónimo de observación participante, de trabajo de campo libre de supuestos y, de ataduras teóricas, de no experimentación, de no cuantificación y que los informes que ella genera son algo "novelesco y anecdótico". Quizás la razón de estas consideraciones es la popularidad alcanzada por uno de los trabajos dé Oscar Lewis: "Los hijos de Sánchez" y el desconocimiento de las elaboraciones teóricas del grupo dirigido por el antropólogo norteamericano.

Estas concepciones han sido desmentidas por numerosos trabajos de investigadores involucrados en estudios enmarcados dentro de estos procesos.

El término etnografía hace referencia tanto a una forma de procedimientos en el trabajo de campo como al producto final de la investigación. Dentro del marco de la Antropología, la etnografía se define como "una teoría de la descripción" que históricamente está vinculada a los relatos de los viajeros europeos sobre las colonias, limitándose, ésta si, a una "mera descripción".

Esto llevó a la convicción de que la etnografía es solo un "reflejo" de la realidad concreta, un dato empírico absoluto, o solo "relativizado" desde las categorías arbitrarias del investigador. Pero el desarrollo histórico de los estudios etnográficos ha llevado a planteamientos bastante más amplios y complejos sobre el significado de esta aproximación en investigación. Wolcott (1975) define la etnografía como la ciencia de la descripción e interpretación cultural. Para Geertz (1973) el etnógrafo debe alcanzar una descripción en profundidad ("thick description"), que debe incluir no solo conductas sino también significados.

En términos generales se considera que la estructura de significados culturales y de las interacciones que los concretizan son resultados de la articulación que dichos significados tienen desde los integrantes de los grupos observados y desde los generados por el etnógrafo.

Esto lleva a considerar el trabajo etnográfico "como una forma particular de "construir el objeto" puesto que no es posible simplemente describir la realidad; ni siquiera describirla como otros la conciben". (Rockwell, E. 1980).

A toda elaboración de la descripción subyacen supuestos teóricos, puesto que no es posible construir "una teoría de una cultura particular", meta de la etnografía, sin tener una teoría general de la cultura.

Por otra parte, al considerar el desarrollo histórico de la etnografía no se puede desligar de la confrontación entre el origen de los etnógrafos y las poblaciones históricamente sometidas a estudio (pueblos primitivos). A esto se agrega el desarrollo del funcionalismo como ruptura con el evolucionismo, que tuvo consecuencias relevantes para el desarrollo de este enfoque de investigación.

Las amplicaciones que esta ruptura tuvo para la etnografía fueron fundamentalmente:

a) Un cuestionamiento a la reconstrucción independiente de las instituciones sociales.

b) La búsqueda de interrelaciones funcionales entre dichas instituciones.

c) La necesidad de establecer el significado de los fenómenos aislados dentro de una estructura. 
d) Un desarrollo amplio de las técnicas del trabajo de campo.

A esto se agrega que a partir de los trabajos enmarcados dentro de la corriente funcionalista se han producido elaboraciones teóricas divergentes, desde la acumulación de conocimientos particulares. (Rockwell, E. 1980).

Debemos considerar también en este proceso histórico de la etnografía el traslado de los estudios de las "sociedades primitivas" a contextos urbanos complejos. Esto ha traído como consecuencia el encuentro con otras disciplinas lo que ha generado las polémicas actuales, fundamentalmente en el área metodológica.

\section{B. El camino etnográfico}

La característica fundamental de los estudios etnográficos, en lo relativo a los procesos metodológicos, es su flexibilidad en cuanto al empleo de las distintas técnicas de recolección de datos e información (observaciones, entrevistas, documentos).

Esto no puede ser confundido con falta de rigor. El rigor de los estudios etnográficos está dado por las reconstrucciones teóricas, por el auto-reconocimiento del nivel en que se encuentra el trabajo y por la búsqueda de coherencia entre las interpretaciones y la realidad.

El proceso de investigación etnográfica se basa en ciertos principios antropológicos fundamentales:

1. Dejar de lado los estereotipos propios acerca de las situaciones estudiadas para explorar como son vistas por los participantes.

2. Convertir lo extraño en familiar, para detectar que lo dado por hecho por investigadores y participantes es, a pesar de todo, extraordinario y así poder cuestionar su existencia.

3. Asumir que para comprender lo particular, se deben mirar las interrelaciones contextuales. Puesto que lo particular es expresión de lo universal.

4. Hacer uso del propio conocimiento de la teoría existente para guiar e interpretar el trabajo de campo.

5. Comenzar la investigación sin categorías específicas de observación, cuestionarios predeterminados o hipótesis precisas. Esto llevaría a una clausura prematura del proceso de descubrimiento de los significativo para el estudio (Wilcox, 1980).

La tarea fundamental de la etnografía es descubrir lo significativo, lo que tiene sentido, lo que es importante para observar. (Ericksson, F. 1977).

El camino etnográfico sigue la ruta de ver más cada vez; de reflexión constante sobre cuerpos teóricos; de asignar significaciones a lo que se oye y se ve; de desarrollar aproximaciones hipotéticas; de redefinición continua. De esta manera una etnografía llega a la interpretación de la realidad que conduce al cuestionamiento y a la reconstrucción teórica. 
Esto implica un compromiso y una participación permanente del grupo de investigaciones en todo el proceso de la construcción etnográfica ${ }^{63}$.

\section{La etnografía llega a la escuela}

Al llegar a estudiar los procesos sociales complejos, los etnógrafos encuentran por un camino natural la institución educativa, como uno de los elementos relevantes de la estructura social.

Como consecuencia de su tradición antropológica los primeros estudios sobre la escuela se enmarcan dentro del campo denominado "micro-etnografía", en razón a que el trabajo de campo se focaliza en la observación de las interacciones, entendidas como conductas verbales y no verbales, al interior de los salones de clase. Las interpretaciones de estos registros tiene su matriz teórica en la socio-lingüística. Su presupuesto básico es, que los estilos de comunicación de la vida cotidiana están culturalmente determinados. En consecuencia, cuando maestros y alumnos provienen de contextos culturales diferenciados, lo que implica no compartir los mismos modos de "competencia comunicativa", se producen "interferencias" en la comunicación y consecuentemente, en las interacciones, afectando a los alumnos.

A partir de estas interpretaciones se intenta explicar el fracaso escolar de ciertos grupos sociales deprivados económicamente. (Rist., R. 1978; Bernstein, B. 1971).

Estos trabajos han enriquecido por un lado, la comprensión de la escuela como instrumento de transmisión cultural y por otro, han llevado a una interpretación más cautelosa de los estudios cuantitativos sobre rendimiento académico. (Ogbu, J. 1980).

Sin embargo, presentan ciertas limitaciones al no considerar la articulación de la realidad escolar, con el contexto social general, pues "en tanto el salón de clase es "la escena de la batalla", las causas de la batalla están en otro lado. Las diferencias comunicativas pueden ser los instrumentos o las armas con las cuales se libra la batalla entre maestros y alumnos. Pero, si queremos descubrir las causas de estas batallas y como eliminar que ocurran y recurran, no progresaremos muchos estudiando los procesos actuales de las batallas y de los instrumentos usados por los combatientes. Necesitamos ir más allá de la escena de la batalla y de sus instrumentos de guerra". (Ogbu, J. 1980).

Esto último intentan los estudios etnográficos globales o "macro-etnografías". Estos trabajos continúan focalizados en la educación, pero consideran también cómo la escolarización está ligada a otras instituciones sociales. Su intento es mostrar e interpretar como la estructura social, incluyendo creencias y valores, se relacionan con las conductas de los participantes en las escuelas.

Los estudios anteriores describen los modelos de interacción entre alumnos y maestros, los tipos de destrezas y conocimientos que los niños adquieren en la escuela y la socialización informal que tiende a reforzar los orígenes sociales de los niños. Para de esta manera, describir e interpretar los modelos de transmisión cultural en términos estructurales. (Grindal, B. 1972; Singleton, J. 1967; Warren, R. 1967).

Además, encontramos la propuesta por parte de John Ogbu de integrar micro y macroetnografía, en una aproximación llamada "ecológico-cultural".

\footnotetext{
${ }^{63}$ Para quienes deseen profundizar más en los procesos etnográficos, consultar. 
Sus presupuestos básicos son:

- La educación formal está ligada con otros factores sociales, especialmente con la economía y con la estructura de oportunidades económicas.

- La naturaleza de esta relación tiene una historia que influye en el proceso actual de escolarización.

— La conducta de los participantes está influenciada por sus modelos de la realidad social.

- Una etnografía adecuada de la escuela no puede limitarse a estudiar los eventos al interior del salón de clase, debe extenderse a las fuerzas históricas y comunitarias relevantes. (Ogbu, J. 1980).

Para lograr esto, Ogbu propone aplicar al estudio de un sistema escolar las mismas categorías que se usan en el estudio de una comunidad: medio ecológico, lengua, economía, organización social, organización política, sistema de creencias, folclor, etc. (Ogbu, J. 1980).

Es dentro de este panorama lleno de contradicciones generadoras de progresos y desarrollos al interior de la discusión teórico-práctica sobre la investigación etnográfica que estamos llevando a cabo nuestro proyecto.

\section{La experiencia etnográfica del proyecto "La Escuela Primaria en Colombia".}

Este estudio es parte integral del proyecto "Escuela y Comunidad en América Latina", en el cual participan además de nuestro país, Bolivia y Venezuela.

La idea de su realización surgió a partir de una revisión de los resultados obtenidos por investigaciones realizadas en algunos países de África, Asia y América Latina sobre la efectividad de los maestros. En la conclusión de este trabajo se sostiene la necesidad de una "investigación diferente" y se propone que tal vez la mejor manera de obtener una visión del proceso educativo y de sus interacciones sea a través del desarrollo y aplicación de técnicas de observación no estructurada, en el marco de la investigación etnográfica. (Avalos, B. y Haddad, W. 1981).

\section{A. Presupuestos teóricos básicos de esta etnografía}

Como dijimos anteriormente, el etnógrafo no parte de una tábula rasa, pues no se puede desconocer el largo camino que ha recorrido la investigación educativa, ni nuestra propia experiencia como educadores. Tampoco podemos hacer a un lado la discusión que se ha dado en torno a la etnografía y que hemos tratado de reseñar en los párrafos anteriores.

En razón a ello, creemos necesario poner en claro cuáles son los presupuestos teóricos que manejamos tanto en lo que tiene relación con los procedimientos investigativos como en lo concerniente al propio objeto de estudio.

Naturalmente, estos presupuestos teóricos guardan un carácter de generalidad, coherente con la perspectiva de investigación adoptada, son ellos: 
-El contexto particular en el cual se realiza la investigación forma parte de una "totalidad" mayor que en alguna medida lo determina. "No se hacen estudios de casos sino estudios en casos

- La dimensión histórica aparece como constante del proceso actual. Se construye un presente histórico a través de la información histórica local y general.

-No se busca coherencia interna en las concepciones, conocimientos de los sujetos, valores y comportamientos. Se intenta interpretar las "categorías sociales" (concretadas en las "claves incidentales") en relación con las condiciones reales en que se producen.

—La educación formal es una institución, que sobrepasa el espacio y la temporalidad de las actividades escolares.

- La escuela es una institución de la sociedad civil, donde se concretiza la relación pedagógica que media la transmisión de saberes y prácticas sociales.

—La escuela y la comunidad no son elementos separados y diferenciados; son una identidad con responsabilidades y actividades distintas.

-El fracaso escolar aparece en relación a los procesos de legitimación de la escuela y consecuentemente al sentido que la misma tiene en sociedades históricamente determinadas*.

A partir de estos presupuestos teóricos hemos planteado un conjunto de preguntas directrices de nuestro estudio: ¿qué sucede en la escuela?; ¿qué se enseña?; ¿cómo se enseña?; ¿qué se aprende?; ¿por qué se aprueba?; ¿por qué se fracasa?; ¿por qué se van los niños de la escuela?; ¿por qué se quedan?

\section{B. Lo concreto de la etnografía: el trabajo de campo}

El trabajo de campo está centralizado en cinco escuelas públicas ubicadas en el área urbana y en el área rural. Las escuelas fueron asignadas a través de contactos establecidos con las Secretarías de Educación y en acuerdo con los supervisores encargados de las zonas. Están ubicadas en Ubaté, Zipaquirá, Chía, Sesquilé y Bogotá, D.E. En algunas de ellas, se están llevando a cabo innovaciones curriculares: Programas de Escuela Nueva y Centros Experimentales Pilotos.

Al iniciar nuestro estudio realizamos reuniones con la totalidad de los maestros de cada plantel, explicándoles con claridad cuáles son los propósitos de nuestro estudio y las formas de trabajo que él implica.

Los maestros, inicialmente, mostraron cierto recelo frente a la posibilidad de nuestra presencia casi permanente en la escuela, y en un caso se negaron terminantemente a participar en el proyecto. Esta es la razón de estar trabajando en cinco escuelas.

Actualmente nuestras relaciones con el personal docente y los niños han logrado el equilibrio y la claridad necesaria para el desarrollo de nuestras actividades de observación

\footnotetext{
* El proyecto internacional tiene como propósito ver cuáles son las formas que adopta el éxito o el fracaso escolar, y el papel que juega el maestro en estas situaciones.
} 
y entrevistas. Esto ha permitido llegar a las relaciones de intimidad imprescindibles con el objeto de estudio, base fundamental de un trabajo etnográfico.

Por otra parte, luego de una primera reflexión sobre las observaciones realizadas en cursos donde se adelantan programas experimentales, vimos la necesidad de participar en algunos de los cursos de capacitación que institucionalmente, se dictan a los maestros. De esta manera intentamos ampliar nuestra posibilidad de interpretación sobre la realidad de estas innovaciones.

Además, desde nuestro trabajo en la escuela hemos comenzado a establecer relaciones con los padres de los niños, para extendernos comprensivamente al contexto social en que está articulada la institución escolar. Esta vinculación se ha establecido a través de nuestra participación en reuniones de padres que organizan los maestros. A partir de ellas, hemos podido dialogar con los padres por fuera de la escuela.

Como otro modo de recuperar el presente histórico, hemos entrevistado a algunos niños, sugeridos por los maestros, buscando la visión que ellos tienen sobre la escuela, desde su experiencia de vida.

\section{Los modos de recolección de información}

En el trabajo de campo se ha recurrido a dos modos básicos para la recolección de datos: la observación y la entrevista no estructurada. Vamos a hacer algunas precisiones sobre las mismas y el papel que juegan en nuestro estudio.

\section{La observación}

Este es uno de los procedimientos de recolección de datos que caracterizan los estudios etnográficos. "La observación participante se refiere a la práctica de vivir con los grupos de personas que se estudian, yendo a conocer su lenguaje y sus formas de vida a través de una interacción intensa y continua en la vida cotidiana. Esto significa conversar con la gente, trabajar con ella... estar presente en tantas situaciones como sea posible, aprendiendo a conocerlos en tantas situaciones y formas como se pueda... también es importante estar alerta a cualquier información que aparezca, para seguir y comprender cualquier evento o hecho que no ha sido anticipado o que parezca inexplicable. Las formas de realizar ésto son a menudo sutiles y difíciles de definir". (Berreman, 1968).

Los procesos de observación son selectivos, aunque esta selectividad en muchos casos es inconsciente, relacionada con la visión de la realidad y las concepciones teóricas del investigador.

"La tarea de observación etnográfica no procede de un momento en que se "ve" todo a otro en que se seleccionan o definen cosas específicas que observar, sino al revés; inicialmente se observa poco, por el proceso inconsciente de selección y es necesario entrenarse a "ver" más. Esto se logra por un lado mediante la apertura a "detalles" que aún no encajan en ningún esquema, y por otro lado, mediante las señales que proporcionan quienes participan en el contexto, que indican nuevos elementos y relaciones significativas". (Rockwell, E. 1980). Los procesos de observación* en nuestro trabajo

\footnotetext{
* Estas referencias a la observación en nuestro trabajo ya han sido publicadas en el Informe de Avance del proyecto, de circulación restringida.
} 
se realizaron inicialmente al interior del salón de clase, completándose la información aquí recogida con las entrevistas que se efectuaron con maestros, niños y padres.

En el desarrollo del trabajo de campo percibimos que restringiendo nuestras observaciones al aula, dejamos por fuera muchos de los eventos que sucedían al interior de la escuela, tales como actos escolares, la entrada y salida de la escuela, el recreo, las reuniones con padres y las reuniones de maestros. En razón de ello, extendimos nuestras observaciones a estos eventos escolares, que también aparecen como significativos tanto para los maestros como para los niños. Pensamos que las observaciones nos permiten conocer con cierta objetividad la vida cotidiana de la escuela, ya que son realizadas en forma más o menos sistemática y continua.

\section{La entrevista**}

Consideramos que la entrevista es uno de los instrumentos fundamentales para la recolección de información primaria. Las entrevistas tienen como propósito reconstruir historias de vida de maestros, niños y padres, así como también opiniones de la administración sobre la escuela, para articular esta información con la recogida a través de las observaciones.

No se pretende presentar una historia de vida de los maestros, padres y niños tan minuciosa que abarque el ciento por ciento de los acontecimientos de su vida; pero sí que dicha historia abarque el ciento por ciento de los hechos importantes que dentro del transcurrir de la vida puedan tener significado frente a la educación.

En el caso de los maestros, permite analizar su posición dentro de la educación, la escuela, la comunidad, su discurso pedagógico, sus vivencias familiares y sociales y la posible relación que todo esto tenga con su practica pedagógica.

Se emplea como parte del estudio porque a través de ella se puede llegar a un contacto personal y directo con los sujetos que son parte de la investigación y crear el "rapport" que les permite decir lo que piensan y sienten libremente, con su propio lenguaje que es parte de su realidad.

La anterior consideración se fundamenta en el hecho de que la relación pedagógica no solamente compromete instancias tales como profesor-alumno, alumno-escuela, sino que allí se hayan comprometidas muchas más determinaciones que remiten y develan una esfera más amplia, donde la totalidad social ofrece mayor contenido y universalidad.

En cuanto a los profesores se refiere, están siendo entrevistados sin más criterio de selección que su aceptación e interés por participar en el estudio. La duración de las entrevistas han sido en promedio dos horas, con una variación de veinte minutos a una hora.

Los niños entrevistados, se han elegido por el conocimiento que de ellos tienen los investigadores y en el asesoramiento de los profesores. En relación con los padres de familia, las entrevistas se organizan con los padres de los niños seleccionados.

\footnotetext{
${ }^{* *}$ Este aparte está publicado en el Informe de Avance del Proyecto, de circulación restringida.
} 
Abordar el objeto de investigación hasta el momento nos obliga a ocupamos de estas distintas perspectivas, en tanto éstas conforman una fuente de extraordinaria riqueza para el proyecto en general.

Con el fin de evitar, o al menos disminuir, el carácter artificial y rígido usual en las entrevistas, se desistió de emplear un cuestionario. Estas se desarrollan hasta donde es factible, partiendo de las explicaciones dadas en un comienzo sobre el tema, aspectos y puntos a tratar; pero ya que no se podía esperar que todos los sujetos abordaran espontáneamente todos los aspectos posibles del tema sin preguntas ocasionales, las entrevistas se realizan con ayuda de una pauta, que permite hacer preguntas cuando estas se refieren a las opiniones manifestadas por el entrevistado, repetir preguntas con otra formulación cuando un tema no parece agotado, omitir preguntas si éstas se responden por sí mismas, ubicar al sujeto con el avance cronológico de la entrevista, ser flexibles y abarcar la totalidad de los temas.

La flexibilidad se extiende también a que la pauta utilizada puede ser completada y modificada en el curso de la investigación. La razón que obliga a tener esta flexibilidad en la entrevista y por otro lado, la obligación de profundizar, aparece en el momento en que encontramos una cierta diversidad de contenidos entre lo significativo de la entrevista y las claves incidentales de la observación de clase.

La pauta de la entrevista no debe considerarse como una lista de preguntas preestablecidas y obligatorias, sino que se debe entender como una ayuda-memoria.

Las entrevistas han sido grabadas y transcritas a la letra. No ha habido rechazo; pero, en algunos casos se han tenido que disipar dudas en relación a la grabación de la entrevista.

\section{Contenidos y formas de la interpretación}

Uno de los momentos más críticos en el proceso de investigación etnográfica es el de la interpretación de los datos e informaciones recogidas durante el trabajo de campo. Es por esta razón que presentamos nuestras reflexiones sobre este problema, iniciando con algunas aclaraciones que creemos imprescindibles sobre este paso del desarrollo investigativo y mostraremos en un ejemplo lo que planteamos como interpretación.

Es normal esperar en la actitud natural denominada sentido común que la interpretación sea una opinión más. Interpretar supone en otros ámbitos categorizar una realidad; categorización que se encuentra estrechamente ligada a un modelo. En este caso interpretar es entendido como el logro de coherencia entre cierta particular categorización y su lugar en la estructura. No podemos descartar las implicaciones que esta normatividad genera en los ámbitos técnicos y metodológicos. Creemos que este tipo de intentos normativos llevan a una extrema rigidez y dogmatismo que estaría contradiciendo el "pathos" por el conocimiento que guía toda investigación.

Desde tiempos remotos en el intérprete quien situado en medio, permite la comunicación, la cual solo es posible si el intérprete plantea y sostiene una relación significativa, y si esta comunicación es necesaria para quienes la solicitan.

El intérprete es el portador de una capacidad que solo se logra cuando se ha estado inmerso y se posee experiencia en, por lo menos, dos sectores de la realidad. 
Desde esta óptica, el intérprete y toda interpretación, como labor mediadora que relaciona los eventos, los hechos y las representaciones, lleva a la disolución del dogmatismo de los modelos y abre un espacio de trabajo donde el objeto de la investigación se muestra y se construye.

La necesidad que tiene toda investigación con vocación de realidad es interpretarla. Por otro lado la interpretación como instancia mediadora podrá lograr su enriquecimiento y concreción si la actividad y el trabajo investigativo accede a la intimidad de lo investigado.

Por lo tanto, interpretar, ser intérprete en una investigación es lograr experiencias y relacionarlas.

La experiencia que el intérprete adquiere es la de relacionar lo particular con lo universal y en cada caso la relación de estos conocimientos al interior del proceso histórico-social.

\section{Relación observación - interpretación}

Es una costumbre generalizada opinar que en toda observación el evento observado entrega su contenido en su más pura inmediatez. Esto implica desconocer la actividad que posee el observador como intérprete, como mediador, que en cada situación percibe selectivamente. Por lo tanto es tan necesario observar como conocer el ángulo selectivo de quien observa. Además, ni la observación, ni la capacidad universal de distinguir son condiciones naturales, pues guardan una íntima coherencia con el grado y clase de relación que el observador posee con el evento observado.

Lo anterior permite reflexionar sobre los diferentes niveles de observación y sobre las diversas posibilidades de selección, interpretación y conocimiento.

El contenido de la observación es, entonces, una síntesis, pues depende en cada caso de la relación observador-observado. Esto explica porqué ante un mismo evento, grupos humanos y aún personas, refieran representaciones distintas y en muchos casos, grotescamente, opuestas.

Por otro lado, el contenido de la observación no solo remite a la relación del observador, sino que también depende de la historia misma del objeto, puesto que éste es una síntesis de la actividad en una sociedad históricamente determinada.

En consecuencia, cuando se observa y se supone que el contenido del objeto está en el objeto mismo, se olvida que sus determinaciones fundamentales están en sus génesis, y ésta en las relaciones históricas.

Captar la objetividad implica un doble proceso: por un lado el de la observación que en cada momento supera los límites del anterior permitiendo ver más y por otro, el proceso de la interpretación que en cada momento permite enriquecer la teoría. Cada momento es una unidad en sí mismo, y como tal adquiere una relativa independencia. Es en sí mismo una estructura significada y significativa.

Estas unidades aparecen en el trabajo investigativo y deben ser discriminadas desde los niveles más elementales hasta los más complejos, mostrando como sus límites guardan una estrecha relación. 
Las unidades iniciales de este proyecto son el resultado del trabajo de las observaciones de clase en notas ampliadas y de las formalizaciones que se presentan a continuación.

\section{Un empleo de observación: posibles modos de análisis e interpretación}

\section{Aclaración necesaria}

Los registros observacionales los realizamos de manera continua, considerando períodos de tiempo, por ejemplo desde el punto de vista de la organización de la clase por parte del maestro. Su duración promedio es de cuarenta minutos, tiempo en el cual se desarrolla una actividad completa en el aula.

Las observaciones son realizadas por dos personas del grupo. Se recogen las notas iniciales y luego se elaboran las transcripciones ampliadas, para evitar sesgos en el trabajo y poder obtener una visión más completa de los acontecimientos observados, generalmente se recurre al uso de una grabadora para lograr una mayor textualidad en los registros verbales, pero nunca se la considera un sustituto de nuestra presencia en el evento observado.

Hemos acordado cierto tipo de notaciones para indicar algunas situaciones, tales como:

/ I -conductas no verbales

" " - -registro textual

' ' - -registro casi textual

..... - -falta parte del texto

M -maestro

( ) -comentarios del observador

$A_{\circ} \quad$-alumno

$A_{a} \quad$-alumna

Algs. $A_{o s}$-algunos alumnos

Curso 5to.*

11:00 Nos dirigimos al curso quinto; los alumnos ya están en el salón esperando a la profesora. Nos sentamos en la parte de atrás del salón, en una banca ocupada por los niños. Mientras esperábamos a la profesora, observamos en qué se ocupaban los niños: trabajaban calcando dibujos de los huesos de la cabeza, coloreando

* Por razones de respeto a los informantes y a los observados no se dan datos ni sobre el lugar ni sobre la fecha de realización de la observación. 


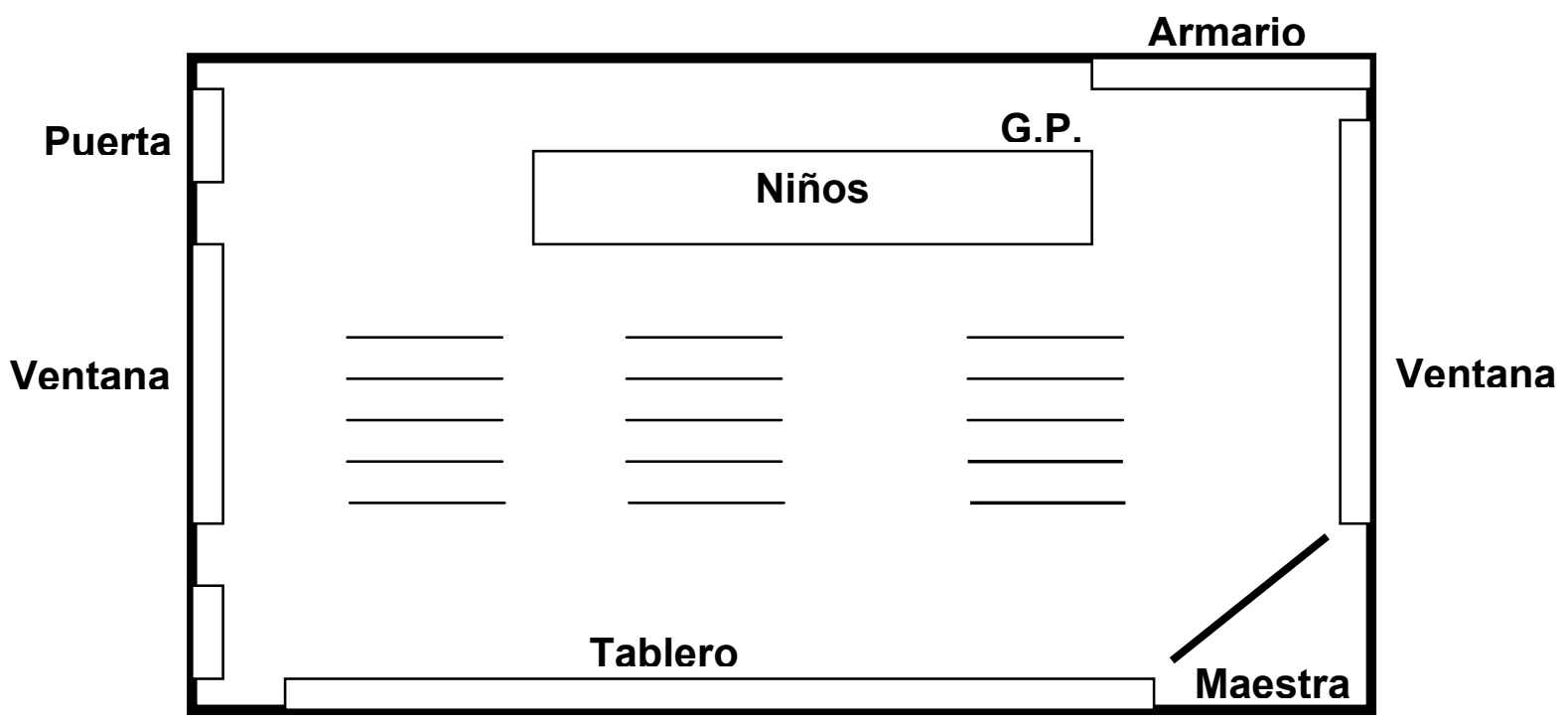

A y colocando nombres. La situación del salón es la siguiente: frente al tablero hay tres filas de pupitres ocupadas por las niñas y al final del salón una mesa grande antigua, con una banca compartida por los cinco niños.

11:05 Se acerca la Directora y dice:

"la profesora no demora, es que salió a traer unos libros; ya viene"

Preguntamos qué clase tienen ahora?

Aos: $\quad$ Clase de ciencias.

A。: /de pie/ "llegó la señora Isabel"

Nosotros preguntamos: quién es la señora Isabel?

Ao: "la profesora"

11:12 No ha comenzado la clase, la profesora (Sra. Isabel) se acerca al otro curso quinto. Habla con la otra maestra; le entrega un paquete. La Directora y otros profesores se acercan y conversan.

A Los alumnos se paran y conversan entre sí. Algunos se dirigen hacia el cronograma que está sobre la pared, miran y se devuelven a su sitio.

11:13 /entra la profesora por la puerta de atrás, con unos materiales, (libros azules), comienza a llamar a los niños por la lista/

"Vamos a entregar los libros.... ¿quién más? Flor - Gladys Marta. Los marcan y los forran.

/la maestra saca libros del paquete y los va entregando/

M: “¿ninguno más había pagado? quedan poquitos libros allí y siempre se economizan treinta pesos".

¡un alumno se levanta va hacia la profesora y le lleva la plata!

A $\quad$ : $\quad$ "a la hora de entregarme. Le toca esperarse hasta mañana

..... Cómo se llama el niño?" 
G: $\quad$ suspende registro y observa a una niña que le muestra a otra el libro de Lenguaje de quinto que acaba de entregar la maestra. (lo hace con cierta picardía). Es una lámina que presenta una escena de la conquista donde unos españoles llevan a unos indígenas hacia una casa colonial. Los indígenas van semi-desnudos.

B /la maestra sentada, desde su escritorio, pide la tarea/

M: "Su tarea de Ciencias Naturales"

/la maestra mira un texto y otro como buscando algo/

a) /los niños buscan y sacan sus cuadernos de Ciencias/ "¿qué era la tarea?"

"la cabeza y el cuerpo humano"

M: “¿ya? bueno, se ponen de pies. Ustedes se acuerdan de esta canción de la Batalla del Calentamiento?"

As: $\quad$ la coro/ sí!

M: Bueno, la van a cantar. Con ánimo para que se calienten”.

/la maestra va caminando al fondo del salón, hacia el rincón del material y saca una calavera/

/los niños cantan en tono muy bajo y (perezosamente)

M: $\quad$ (tono de regaño) "Eso qué es? Quée, están dormidos? A ver, con ánimo!"

b) /la maestra se dirige nuevamente al frente del salón/

M: /dando instrucciones sobre el canto/ "Con todo el cuerpo. A ver, saltando" (los alumnos casi gritan)

(se desordena un poco el curso)

M: "Bueno, ahora sí, sentaditos". "Con ese canto se calentaron y además.., qué más hicieron?"

$A_{s}: \quad$ la coro/ "movimientos"

1ab) $\mathrm{M}$ : "con qué hicieron movimientos?" ¡los alumnos responden indistintamente!

As: "con la cabeza, con el cuerpo, con las extremidades"

"Y después, con qué?"

$\left.1 a^{*}\right)$ Alg. $A_{s}:$ "Con todo el cuerpo"

M: "Bueno, a ver, tienden una tareíta. En qué consiste esa tarea? A ver, Elvia..."

Elvia: "los huesos de la cabeza"

M: Cuáles son esos huesos de la cabeza? Porque a mí sí, ya se me olvidaron totalmente"

/los alumnos comenzaron a contestar en desorden/ 
M: "Una sola. A ver"

$A_{a}: \quad$ "Frontal"

M: "Pero, me los va mostrando. A ver"

/la alumna señala en la calavera/ (aparenta duda al señalarlos, nombrarlos)

Aa: "Sí, señora"

M: $\quad$ tem..."

$A_{a}: \quad$ temporales"

M: "ра..."

$\mathrm{A}_{\mathrm{a}}: \quad$ "parietales"

M: "le faltan dos, que esos sí no los podemos tocar porque están adentro. A ver. ¿Cómo se llaman esos dos?

$\left.1 a^{*}\right) \quad$ /los alumnos, en desorden, responden esfenoides y con dificultad pronuncian etmoides, luego que la maestra lo ha dicho/

M: "esos deben estar dibujados en el cuaderno con los nombres respectivos

A: La mía señorita!" (hace referencia a su tarea)

/la maestra se dirige a un niño/

M: "de qué vale una tarea si no tiene nombres. Póngale los nombres, para distinguirlos"

/la maestra se dirige ahora a una niña/

M: $\quad$ "A ver, no hizo la tarea. No hizo la de Historia y vamos a ver la de Ciencias y tampoco!" (tono de regaño)

/la maestra se dirige a otra niña/

M: $\quad$ "Esa letra tan terriblemente, mira!"

/la maestra al llegar a la mesa donde están sentados los

niños los recrimina por no hacer la tarea/

/la maestra tiene un esfero rojo con el que va revisando la

tarea; va revisando uno a uno/

/un alumno ubicado al lado del observador dice:

1a)

$A_{0}: \quad$ "no la hice. Mañana la traigo"

/un compañero del anterior le responde/

$\mathrm{A}_{\circ}$ : $\quad$ "ya para qué?"

"Pero les dije: la pueden sacar de las Ciencias, la pueden dibujar, la pueden pasar".

/la maestra se dirige a un alumno/ 
M: "no la hizo, usted no me ha hecho tareas. ¿dónde está la tarea? /la maestra dirigiéndose a todo el grupo dice:

M: "los niños son los más perezosos para hacer tareas".

/P. Suspende registro para mirar como la maestra califica la tarea: coloca una B en las tareas completas/

1a) M: "ahora sí guarden los cuadernos. Me hacen el favor los niños de ser más cumplidos con sus tareas".

A: "Yo si la hice"

(la maestra ignora la afirmación)

M: "La mayoría de las... de los niños... pudiendo sacar de unas ciencias, sacar la lámina o comprar lámina y pegarla. Era una cosa supremamente fácil $y$ no la pudieron hacer. Los niños tienden a ser muy perezosos. Bueno, pero de ahora en adelante, van a ser los mejores. Yo creo, porque eso queda como muy mal". "Pase Daniel y nos dice los nombres de los huesos de la cabeza". /Daniel pasa y ayudado por la maestra los va diciendo. Luego se sienta/

M: "Una niña para que se nos vaya grabando de tanto repetirlos aquí"... /la maestra le presenta la calavera para que vaya señalando los huesos

M: "mírela bien, sin miedo. Esos dos remienditos que tenemos aqui" / la maestra señala en la calavera/ (la niña asustada evita tocar la calavera)

M: "Vuelva y me los dice bien. Está toda equivocada... et, et, et..."

$\mathrm{A}_{\mathrm{a}}$ : "moides"

/la niña se sienta/

M: "Gladys, venga, tenemos que aprenderlo muy bien, para toda la vida... No diga frontales que no tenemos sino uno". "A ver, otra de las niñas. Pase Janeth" /Janeth pasa al frente y repite los nombres de los huesos, señalados por la maestra en la calaveral

M: "Bueno, todas. Yo creo que ya todos se los saben. Bueno, me los van a repetir".

/la maestra va señalando los huesos sobre la calavera/

M: "Este?"

$A_{\circ s}:$ /a coro/ "temporal" 
M: "Este?"

$A_{o s}:$ /a coro/ "frontal"

M: "Estos dos?"

$A_{o s}:$ /a coro/ "temporales"

M: "Este?"

$A_{o s}: \quad$ la coro/ "occipital"

M: "Y los dos de adentro?"

$\left.1^{a}{ }^{* * *}\right) A_{o s}:$ la coro/ "etmoides y esfenoides"

M: "estos son los huesos de qué...?"

$A_{o s}: \quad$ la coro/ "la cabeza"

M: "De la cabeza. Pero bueno, ahora una niña me va a decir de qué está formado un hueso. A ver, de cuántas partes se compone un hueso? Alba"

Alba: "De tres".

M: Bueno, no diga el número, sino vaya diciéndome... A ver una parte qué?"

Alba: "tejido esponjoso"

M: "De una parte interna y una parte externa. Bueno, a ver la parte interna es como...? Eso que me dijo"

Alba: "tejido esponjoso"

M: "Muy bien, otra... otra partecita"

Alba: "tejido compacto"

M: "tejido compacto", “¿, qué más?" "Una sustancia que le da vida al hueso, cómo se llama?"

Alba: "médula"

M: "médula, muy bien!" "De qué más está formada? A ver, otra cosita" /no entendemos la respuesta de Alba/

$\left.1 \mathrm{a}^{* * *}\right) \mathrm{M}$ : "Bueno, eso es el componente ¿no? De lo que está formado...." "Los huesos de la cabeza como son?, qué forma tienen?

Aos: la coro/ "planos"

M: "Planos, muy bien!". "Será que hay otra clase de huesos"?... Fuera de planos? 
$\mathrm{A}_{\circ}$ : "curvos"

(la maestra no se da por enterada de esta respuesta) /dan respuestas de toda clase/

/la maestra al notar que no aciertan, recurre a recuerdos de la clase anterior, donde probablemente trabajaron sobre otra clase de huesos/

M: "Los que vimos ayer, cómo eran?"

Aos: la coro/ "largos"

M: "Y los otros?"

Aos: /a coro/ "cortos"

M: $\quad$ "Pero, estos de la cabeza, cómo son?"

Aos: la coro/ "planos"

M: "Hoy, qué huesos iremos a ver?

A1.As:"Los de la cara"

M: "Los de la cara" (tono de reafirmación)

/la maestra tiene la calavera en la mano y va señalando/

M: "Eso si es muy fácil porque todos deben... claro que aquí nos falta una partecita" /señala una parte de la calavera/ "no la vamos a poder mirar aquí muy bien, pero nos vamos a hacer la idea". "A ver... Estos dos aquí como se llamarán". "A ver, yo creo que ustedes saben si necesidad, sin necesidad, de decirles van a saber.., cómo se llamarán estos dos?"

/la maestra está ubicada al frente de la clase entre dos filas de bancos/

A1.As:"Pómulos"

(la maestra no escucha esta respuesta)

M: "no, miren aquí". "Cuando están corriendo se ponen coloraditos, cómo se llamarán?"

/los alumnos en voz más alta dicen en coro

Aos: "pómulos"

M: "pómulos" "Muy bien!" "Estos dos como se llaman?" /la maestra señala sobre la cara de una niña/

Aos: /a coro/ "Pómulos"

M: "Este de aquí, con el cual... se puede mover para abrir la boca y cerrarla, ¿cómo se llamarán estos dos?"

Aos:...

M: $\quad$ "Bueno, así es el nombre... cómo les digo yo...? 
Aos: "Vulgar"

M: "Vulgar, pero el nombre propio como se llamará?"

II A1.As:"Maxilar"

M: “¿Cómo?"

(esta pregunta genera duda y son menos los alumnos que responden)

A1.As:"maxilar"

M: "Maxilares", "maxilar qué?"

A1.As:"maxilar superior e inferior"

/la maestra indica sobre la calavera los huesos maxilares/

M: "Bueno, entonces vamos a repetir los que ya me han dicho. Estos dos..."

/la maestra señala sobre la calavera/

Aos: a coro/ "pómulos"

M: "estos dos?"

Aos: /á coro/"maxilares"

M: "estos que forman la nariz.., pero cómo se llaman estos dos que forman la nariz?" (tono de disgusto) "Eso es fácil, la misma palabra lo dice".

A1.As: "nasales"

M: "muy bien! Cuáles?"

A1.As: "nasales"

M: "vamos escribiendo.., para saber cuántos huesos nos van a dar" /la maestra coloca la calavera sobre el escritorio y se dirige al tablero/

II M: "Dos qué?"

Aos: /a coro/ "Pómulos"

M: "Dos qué...?"

Aos: /a coro/ "Maxilares"

M: "uno qué...?"

/la maestra va escribiendo en el tablero/

2 pómulos

2 maxilares: superior - inferior

2 nasales

2 cornetes 
A1.As: "nasal"

(la maestra no se da por enterada de esta respuesta)

M: "uno superior y uno qué...?"

Aos: la coro/ "inferior"

M: "Qué más, cuáles otros huesos hay?"

Aos: la coro/ "nasales"

M: (tono reafirmativo) "dos nasales" "A ver, qué más?"

"Vayan mirando y me van diciendo y vamos contando a ver cuántos huesos van"

/los alumnos no responden/

M: "Cuál otro? A ver, cuál será? A ver, esos que forman la nariz, también; que hay veces cuando ustedes tienen catarro se les tapa y hablan gangositos, cómo se llamarán esos dos?

/la maestra hace una indicación hacia el fondo del salón/

M: "A ver, allá" "Ustedes están oyendo? En la casa cuando se les inflama, allá..."

A1.As: "gotas nasales"

M: $\quad$ "No, eso es lo que se echan allá para que se les desinflame; pero cuando a ustedes les da catarro por la noche, van a dormir y no pueden porque se les tapan los..., los qué? A ver"

Ao: "bronquios".

M: "No! No!" "Aquí, en la nariz; dos huequitos que tenemos allá por dentro, cómo se llamarán... los.., los...?” ¡los alumnos no responden!

M: "los cornetes" "A ver, vamos otra vez, vamos repitiendo. A ver los huesos de la cara son...

/un niño de la última banca, al copiar del tablero lo que escribe la maestra deletrea: "I-o-s-c-o-r-n-e-t-e-s" /los alumnos van leyendo al indicar la maestra lo escrito en el tablero; lo hacen en desorden/

M: "A ver, qué más, qué otros? Van escribiendo. Un momentico. Un momentico que aquí nos llaman...

/la maestra se dirige hacia la puerta/

/una niña por la ventana llama a la profesora y dice:

Aa: "dígale a la profesora que la necesitan"

/la maestra regresa al frente de la clase/

M: "Voy a poner un trabajito, mejor... Tarea de lenguaje, mejor... mientras ahora terminamos". "Con estas palabras, van a relacionarlas y a hacer un cuentico" 
/la maestra escribe en el tablero/

M: (tono de dictado) "Campo, como la mayoría viven en el campo les queda fácil”.

M: "Se van a estar juiciosos. Ahí quedan cuidados"

(hace referencia a los observadores)

\section{Campo-animales - río - casita - abuela - niños árboles - cielo azul - paisaje.}

Una primera forma de análisis de las observaciones es un proceso que llamamos de formalización. Este consiste en considerar los pasos fundamentales que sigue la actividad desarrollada en el aula y que surgen de una lectura y relectura de notas ampliadas, como las que acabamos de transcribir más arriba.

Cada momento se anota en la sucesión cronológica en que se da en la realidad. De las notas ampliadas que aquí presentamos surge una formalización que separa a la actividad en dos grandes momentos:

A-Descripción del contexto

B- Manera de dictar clase

La formalización completa es la siguiente:

A-Descripción del contexto - entrega de material

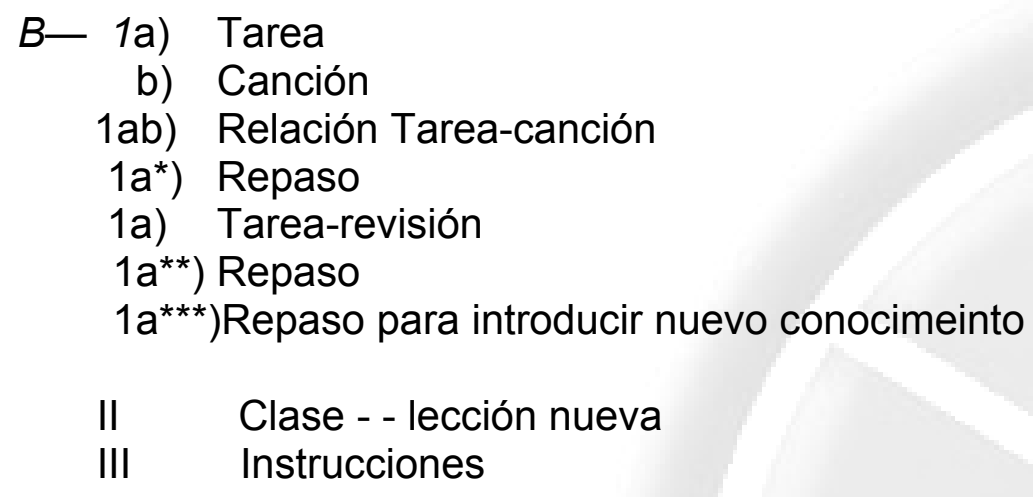

Podemos comparar esta formalización que nace de la realidad de la observación de una clase, con el modelo generalizado que encontramos en los textos y manuales de Didáctica que se recomiendan para la formación y capacitación de docentes. Este modelo presenta los siguientes pasos metodológicos, a los que vamos a agregar algunas situaciones concretas de ejemplificación de acciones posibles, para ser llevadas a cabo en la ejecución

I - Introducción 
A manera de introducción el maestro puede revisar tareas, realizar juego y canciones, desarrollar una conversación sobre el tema que se va a tratar, como forma de motivar la actividad de los alumnos.

II - Desarrollo de la clase

Este puede estar centrado en la sola exposición del "maestro o en una relación expositiva - participativa.

III - Conclusión

Puede hacer ejercicios de aplicación y posteriormente asignar tareas o lecciones.

Parecería bastante claro que ambas cronologías de momentos de la clase están atravesadas por la tradición pedagógica más ortodoxa, nacida en la "Pedagogía General" de Herbart, y que se ha mantenido con pequeñas variaciones como el modelo fundamental del quehacer pedagógico.

La matriz teórica de la clase observada es esta ortodoxa didáctica, aunque el maestro no sea consciente de este origen de su quehacer. De todas maneras en la realidad, estos momentos o pasos didácticos se encuentran matizados y la delimitación entre uno y otro no es lo suficientemente clara.

Para la interpretación de esta primera formalización analítica de la observación vamos a partir de dos preguntas directrices: ¿en qué momentos se articula la realidad con el modelo teórico? ¿Dónde se presentan las contradicciones?

Dejando por fuera lo que hemos denominado "descripción del contexto" que incluye una situación inicial, cuyo contenido real es la entrega de libros comprados por la profesora, vamos a adentramos en la Parte B de la formalización que hemos denominado "manera de dar clase".

La maestra inicia su trabajo con una revisión de tareas, a la que sigue la propuesta de cantar. Hace una vinculación entre los contenidos de la letra de la canción con el contenido de la tarea que debían realizar los alumnos.

M; "con ese canto se calentaron un poquito y además qué hicieron?"

Aos: la coro/ "movimientos"

M: “¿con qué hicieron esos movimientos?"

Aos: /a coro/ "con el cuerpo."

De esta manera, a través de un repaso (nombrar y señalar los huesos de la cabeza y decir cómo están estructurados y qué forma tienen) introduce el nuevo tema (huesos de la cara).

Hasta aquí podríamos afirmar que existe una articulación entre la propuesta teórica y la realidad de lo observado. Comienza entonces el nuevo tema que es introducido por la maestra con la pregunta: "Hoy ¿qué huesos iremos a ver?". Pero este nuevo tema que se 
introduce es claro y evidente que ha sido estudiado por los niños en sus casas. Se inicia entonces una sesión de preguntas y respuestas.

Desde el modelo teórico podríamos sostener que este desarrollo de la clase adopta la forma expositiva - participativa. En tanto, los niños van respondiendo a las demandas de la maestra. Sin embargo, esta participación de los niños apela al recurso de la memorización de los nombres de los huesos de la cara, para finalmente realizar un conteo de los mismos y registrarlos en el tablero.

Habría entonces, un traslado mecánico y nominativo del concepto de participación del alumno, que estaría distorsionando la articulación entre modelo teórico y realidad. Puesto que el significado de participación al interior del primero connota creatividad, imaginación y cuestionamiento, y en la realidad solo apela a la memoria.

Los alumnos han comenzado a consignar en sus cuadernos lo anotado por la maestra durante la sesión de preguntas y respuestas, cuando surge un imprevisto "Un momentico, aquí nos llaman?" y luego: "Voy a poner un trabajito, mejor... Tarea de lenguaje".

De pronto, la clase de anatomía se transforma en tarea de Lenguaje.

Si buscamos la articulación entre modelo teórico y realidad, en el último paso metodológico, encontramos que en el comienzo de la Conclusión lo hubo, pero es claro y evidente que cualquier imprevisto distorsiona esta posible articulación, pues el maestro salta de un área del conocimiento a otra sin considerar las posibles coherencias o incoherencias entre las mismas.

Esta formalización analítica es solo un primer paso de la interpretación de la información recogida durante el trabajo de campo.

Si miramos detenidamente la interpretación guiada por la formalización, parece claro que la misma tiende a rigidizar la realidad, ocultando momentos significativos de los sucesos del salón de clase.

Como dijimos con anterioridad la meta de la etnografía es la búsqueda de lo significativo, del sentido de lo observado. La interpretación presentada anteriormente, sin duda no llega a cumplir con las propuestas de una perspectiva etnográfica en investigación, y no convierte al etnógrafo en intérprete sino en un analista.

Sin embargo, la formalización analítica es un paso imprescindible en la búsqueda de lo significativo, pues sin ella lo significativo aparecería como una simple ocurrencia casual, sin conexiones con su origen: lo observado.

Al interior del salón de clase se producen interacciones que median, no solo contenidos programáticos sino también concepciones y visiones del mundo. Estas se concretizan en ciertos momentos significativos a los que hemos denominado "claves incidentales".

Clave incidental es el momento de la clase en el cual, a través de un diálogo expresado verbal o gestualmente, se median modos de relación con la totalidad social, y éste es el camino hacia la meta de la construcción etnográfica, la búsqueda de lo significativo, del sentido de la realidad, que lleva al fin último de todo proceso investigativo: la construcción de conocimiento. 
En las notas ampliadas encontramos un ejemplo claro de estos momentos significativos que constituyen las claves incidentales.

/la maestra dirigiéndose al grupo dice:

M: "Los niños son los más perezosos para hacer tareas... Ahora sí guarden los cuadernos. Me hacen el favor los niños de ser más cumplidos con sus tareas".

Ao: "Yo sí la hace"

/la maestra ignora esta afirmación/

M: "La mayoría de las... de los niños.., pudiendo sacar de unas ciencias, sacar la lámina o comprar lámina y pegarla. Era una cosa supremamente fácil y no la pudieron hacer. Los niños tienden a ser muy perezosos. Bueno, pero de ahora en adelante van a ser los mejores, yo creo, porque eso queda como muy mal".

El proceso de esta interacción está atravesado por contenidos valorativos de la maestra hacia la posibilidad y efectividad del trabajo de los niños y nos lleva a conjeturar una especial preferencia de la maestra por el grupo de las niñas, en la clase. La consecuencia que aparece inmediatamente es que la valoración mostrada por la maestra a nivel verbal está también vinculada a la ubicación de niñas y niños en el espacio físico de la clase (niñas adelante, niños atrás), en el tipo de pupitre en que trabajan unos y otros, en el hecho de que la maestra en general habla en género femenino. Esto podría estar afectando por un lado las relaciones grupales entre niños y niñas, por sentirse unos privilegiados y otros poco considerados o subvaluados en sus posibilidades de trabajo, y por otro, en las concepciones que los dos grupos generan sobre la escuela que sin duda inciden en los modos y procesos de aprendizaje.

Este tipo de situación concreta, que desde la elaboración y construcción de interpretaciones que sigue nuestro estudio llamamos clave incidental, nos permite ir articulando una interpretación global del papel del maestro, desde el interior del salón de clase, en el fracaso o éxito escolar de los niños, que es uno de los objetivos del estudio que venimos desarrollando.

Por otra parte, creemos que este ejemplo, lleva a clarificar las formas procedimentales en la detección de las claves incidentales, por parte del grupo de investigadores. Además, la articulación de estas claves incidentales lleva en el proceso etnográfico a la elaboración de "categorías sociales" y éstas a la "construcción del objeto" a través de la relación y reflexión permanente y sistemática entre los presupuestos teóricos asumidos por la investigación y la realidad.

\section{Una nota final}

El propósito que animó la redacción de este artículo fue la necesidad del grupo de trabajo de hacer un intento por compartir esta nueva perspectiva en la investigación en educación, que sin dudas, abre nuevos campos de estudio y nuevos modos de aproximarse a ellos.

Además, un interés por señalar los elementos básicos en la construcción de una etnografía mostrando sus límites y posibilidades en la contribución al desarrollo del conocimiento y a la construcción teórica. 
Para finalizar, un comentario de John Ogbu, sobre las condiciones necesarias de un buen etnógrafo: "debe tener sentido de la perspectiva, es decir, ser capaz de distinguir los eventos importantes de los triviales; mantener una aproximación objetiva y escéptica a los datos, tener buen sentido del humor; y tener simpatía, esto es, la capacidad de poder generar una experiencia del mundo como la de los participantes. Todo ello guiado por la comprensión de la naturaleza de la estructura social y de las interacciones sociales en el plano teórico y en la práctica. Esto debe ser acrecentado por el propio proceso etnográfico".

Creemos que entre esta concepción etnográfica y el "mito de lo anecdótico" hay una gran distancia.

\section{BIBLIOGRAFIA}

Avalos, Beatrice; Haddad, Wadi, Reseña de la investigación sobre la efectividad de los maestros en África, América Latina, Filipinas, India, Malasia, Medio Oriente y Tailandia: síntesis de resultados —IDRC_- Ottawa, 1981.

Berreman, Gerald D., Ethnography: Method and Product, en Introduction to Cultural Anthropology: Essays in the Scope and Methods of the Science of Man, James A. Clifton, ed. Houghton Mifflin Co. Boston, 1968.

Bernstein, Basil, Class, Codes and Control, Volumen I, Theoretical Studies Toward a Sociology of Language, Ed. Routledge and Regan Paul, Londres, 1971.

Ericksson, Frederick, Sorne approaches to inquiry in School Community Ethnography, en Anthropology and Education Quaterly, Vol. 2, No. 2 - Mayo 1977.

Geertz, Clifford, The interpretation of Cultures, New York, Basic Books, N.Y. 1973.

Grindal, Bruce, Growing up in Two Worlds Holt, Rinehart and Winston, N.Y. 1972.

Rist, Ray C., Ethnographic Techniques and the Study of Urban School en Urban Lducation No. 10, 1975.

Rockwell, Lisie, La relación entre etnografía y teoría en la Investigación Educativa -D.I.E. -IPN México - 1980 (mimeo)

Singleton, John, Nichu: a japanese school. Holt, Rinehart and Winston, New York, 1967.

Warren, Richard L., Lducation in Rebhause: a gerrnan viliage blt, Rinehart and Winston, N.Y., 1967.

Wilcox, Kathleen, The Ethnography of schooling: implications for educational policy making -California State University/Hayward, julio 1980. (mimeo).

Wolcott, Harry F., Handle with care: necessary precautions lo the Anthropology of Schools en Anthropological perspectives on Education, New York, Basic Books, 1971.

Obgu, John U., School Ethnography: a multi-level approach Department of Anthropology, University of California, junio 1980 (mimeo). 